\title{
Conhecimentos, atitudes e práticas de profissionais do sexo sobre prevenção de infecções sexualmente transmissíveis, antes e após uma intervenção educativa
}

\author{
Knowledge, attitudes and practices of sex professionals about prevention of sexually transmitted \\ infections, before and after an educational intervention \\ Conocimientos, actitudes y prácticas de profesionales del sexo sobre la prevención de infecciones de \\ transmisión sexual, antes y después de una intervención educativa
}

Recebido: 25/08/2021 | Revisado: 31/08/2021 | Aceito: 07/09/2021 | Publicado: 09/09/2021

\author{
Raquel Xavier Guimarães \\ ORCID: https://orcid.org/0000-0001-6038-418X \\ Universidade Estadual Vale do Acaraú, Brasil \\ E-mail: raquelguimaraesponte@ hotmail.com \\ Maristela Inês Osawa Vasconcelos \\ ORCID: https://orcid.org/0000-0002-1937-8850 \\ Universidade Estadual Vale do Acaraú, Brasil \\ E-mail: miosawa@gmail.com \\ Andrea Carvalho Araújo Moreira \\ ORCID: https://orcid.org/0000-0001-9855-1449 \\ Universidade Estadual Vale do Acaraú, Brasil \\ E-mail: dreamoreira@yahoo.com.br \\ Ivaldinete de Araujo Delmiro Gemes \\ ORCID: https://orcid.org/0000-0003-2857-1502 \\ Universidade Estadual Vale do Acaraú, Brasil \\ E-mail: ivaldinete@ig.com.br \\ Juliana Solon Furtado \\ ORCID: https://orcid.org/0000-0001-5447-5982 \\ Universidade Estadual Vale do Acaraú, Brasil \\ E-mail: julianasolon@yahoo.com.br
}

\begin{abstract}
Resumo
Este estudo objetivou avaliar conhecimentos, atitudes e práticas de mulheres profissionais do sexo sobre prevenção de infecções sexualmente transmissíveis (IST), antes e após uma intervenção educativa. Estudo descritivo, transversal, do tipo intervenção, com abordagem quantitativa, realizada com 75 mulheres profissionais do sexo que trabalhavam em ambientes privados na cidade de Sobral - CE. Utilizou-se formulário com variáveis sociodemográficas e de conhecimento, atitude e prática sobre Infecções Sexualmente Transmissíveis (inquérito CAP). A coleta de dados ocorreu nos meses de abril e maio de 2016, sendo as entrevistas transcritas, analisadas e classificadas pelo conhecimento, atitude e práticas, antes e após a intervenção educativa. Os resultados mostraram que as mulheres têm conhecimento adequado sobre como se prevenir em relação às IST, reconhecendo que o preservativo masculino é um dos métodos mais seguros para evitar as IST/HIV. Entretanto, suas atitudes e práticas encontram dissonância ao aceitarem realizar programas sem preservativos por um valor mais alto e não os utilizando com parceiros fixos e cliente preferenciais. Após a intervenção, detectou-se a ampliação do conhecimento sobre saúde sexual. Concluiu-se que o fator financeiro no comércio do sexo sobrepõe ao conhecimento, levando às mulheres a não assumirem atitudes e práticas adequadas sendo obstáculos a serem considerados na promoção da saúde sexual do público estudado.

Palavras-chave: Profissionais do sexo; Mulheres; Infecções sexualmente transmissíveis; Conhecimentos, atitudes e prática em saúde; Enfermagem.
\end{abstract}

\begin{abstract}
This study aimed to assess knowledge, attitudes and practices of female sex workers about prevention of sexually transmitted infections (STIs), before and after an educational intervention. Descriptive, cross-sectional, interventiontype study with a quantitative approach, carried out with 75 female sex workers who worked in private environments in the city of Sobral - CE. A form was used with sociodemographic variables and knowledge, attitude and practice on Sexually Transmitted Infections (CAP survey). Data collection took place in April and May 2016, with the interviews being transcribed, analyzed and classified by knowledge, attitude and practices, before and after the educational intervention. The results showed that women have adequate knowledge about how to prevent STIs, recognizing that the male condom is one of the safest methods to avoid STIs/HIV. However, their attitudes and practices are dissonant when accepting to carry out programs without condoms for a higher price and not using them with fixed partners and
\end{abstract}


preferential clients. After the intervention, an increase in knowledge about sexual health was detected. It was concluded that the financial factor in the sex trade supersedes knowledge, leading women not to assume proper attitudes and practices, which are obstacles to be considered in promoting the sexual health of the studied public.

Keywords: Sex workers; Women; Sexually transmitted infections; Health knowledge, attitudes and practice; Nursing.

\section{Resumen}

Este estudio tuvo como objetivo evaluar los conocimientos, actitudes y prácticas de las trabajadoras sexuales sobre la prevención de infecciones de transmisión sexual (ITS), antes y después de una intervención educativa. Estudio descriptivo, transversal, tipo intervención con abordaje cuantitativo, realizado con 75 trabajadoras sexuales que laboraban en ambientes privados en la ciudad de Sobral - CE. Se utilizó un formulario con variables sociodemográficas y conocimientos, actitudes y prácticas sobre Infecciones de Transmisión Sexual (encuesta CAP). La recolección de datos se realizó en abril y mayo de 2016, siendo las entrevistas transcritas, analizadas y clasificadas por conocimientos, actitudes y prácticas, antes y después de la intervención educativa. Los resultados mostraron que las mujeres tienen un conocimiento adecuado sobre cómo prevenir las ITS, reconociendo que el condón masculino es uno de los métodos más seguros para evitar las ITS / VIH. Sin embargo, sus actitudes y prácticas son disonantes al aceptar realizar programas sin condón por un precio mayor y no utilizarlos con socios fijos y clientes preferenciales. Tras la intervención, se detectó un aumento en el conocimiento sobre salud sexual. Se concluyó que el factor económico en el comercio sexual reemplaza al conocimiento, llevando a las mujeres a no asumir actitudes y prácticas adecuadas, obstáculos a ser considerados en la promoción de la salud sexual del público estudiado.

Palabras clave: Trabajadores del sexo; Mujeres; Infecciones de transmisión sexual; Conocimientos, actitudes y prácticas de salud; Enfermería.

\section{Introdução}

A prostituição, conhecida pelo senso comum como a "mais antiga profissão do mundo", sempre foi um tema envolto por muita polêmica, tanto nacional como internacionalmente, de modo que, historicamente, já se discutiu o combate à prostituição por conta da sífilis; com o advento do HIV/Aids trouxe temores e práticas sociais discriminatórias em relação à prostituição e mais recentemente, no Brasil, debates encontram-se divididos entre sua proibição ou regulamentação (Figueiredo \& Peixoto, 2010).

A ideia de risco gerou mais preconceito e discriminação e mostrou os limites de suas intervenções para conter a epidemia. A história da Aids no Brasil aponta para a importância de incluirmos os direitos humanos na construção de nossas estratégias de prevenção. O direito de ir e vir, de expressão, social, sexual, das pessoas vivendo com HIV/Aids devem ser considerados ao fazermos prevenção. Precisamos lutar pela garantia destes direitos! A ideia de vulnerabilidade leva em conta se exercemos ou não esses direitos em nosso dia-a-dia. Ela afirma que o contexto influi diretamente na exposição dos grupos populacionais ao HIV/Aids (Brasil, 2008).

O exercício da prostituição impõe comportamentos que as tornam vulneráveis à aquisição de infecções sexualmente transmissíveis (IST) e o vírus da imunodeficiência humana (HIV), tais como: elevado número de parceiros sexuais, mantendo um grande número de coitos por dia, relações sexuais desprotegidas, além dos eventuais micros traumatismos vaginais, com provável perturbação do ecossistema vaginal, acrescida do risco decorrente do uso de substâncias químicas, como consumo de drogas ilícitas e de bebidas alcoólicas, assim como histórico de encarceramento, baixo nível educacional, barreiras em relação ao gênero e a marginalização socioeconômica (Aquino et al., 2010).

A vulnerabilidade é trabalhada quando se procura entender os contextos em que os sujeitos, objetos da investigação, estão inseridos: suas condições de vida e de trabalho, a intersubjetividade e autorrepresentação, suas formas de organização e de socialização, bem como o grau de "empoderamento" das mulheres para garantir seus direitos mínimos como cidadãs e profissionais (Brasil, 2003).

É notório que o perfil epidemiológico das IST vem se modificando durante as últimas décadas em todo o mundo, com aumento expressivo do número de casos entre mulheres. Os contextos históricos revelam que as mulheres brasileiras enfrentam obstáculos em virtude do preconceito, dos processos vinculados à dinâmica de gênero e das relações sexuais e reprodutivas. A multiplicidade de parceiros sexuais, assimetria nas relações entre mulheres e homens, dogmas religiosos e implicações morais 
expõem o público feminino às IST e, concomitantemente, acentua a vulnerabilidade das mulheres (Moura et al., 2021; Nascimento et al., 2017).

Estudos têm mostrado taxas elevadas de positividade para IST em mulheres em situação de prostituição. Em uma metanálise, realizada com 99.878 mulheres profissionais do sexo de 51 países, identificou-se prevalência para o HIV de 11,8\% (Silitonga et al., 2011).

No Brasil, de 1980 até junho de 2020, foram registrados 664.721 (65,7\%) casos de aids em homens e 346.791 (34,3\%) em mulheres. No período de 2002 a 2009, a razão de sexos, expressa pela relação entre o número de casos de aids em homens e mulheres, manteve-se em 15 casos em homens para cada dez casos em mulheres, em média. No entanto, a partir de 2010, observa-se um aumento na razão de sexos, que chegou a 23 casos de aids em homens para cada dez casos em mulheres em 2017, razão que se manteve em 2018 e em 2019 (Brasil, 2020).

Um estudo multicêntrico realizado no Brasil com 2.523 mulheres em situação de prostituição de nove Estados e do Distrito Federal verificou-se prevalência de 4,9\% para o HIV e de 2,5\% para sífilis (Szwarcwald, 2010). Em São Paulo, pesquisa desenvolvida por Pogetto et al. (2011) identificou a prevalência de IST entre prostitutas em um município de médio porte do interior paulista no ano de 2008. Considerando uma amostra de 102 mulheres em situação de prostituição, a prevalência geral das IST foi de 71,6\%. Considerados isoladamente e em associação, os maiores valores encontrados foram: HPV $(67,7 \%)$ e infecção clamidiana (20,5\%). A tipagem do HPV evidenciou genótipos oncogênicos. A prevalência de sífilis foi de $4,0 \%$ e de tricomoníase $3,0 \%$. Conclui-se que a prevalência de IST foi elevada, pois, aproximadamente $2 / 3$ das mulheres, apresentavam alguma doença assim classificada.

Diante deste contexto epidemiológico e de vulnerabilidade, intentou-se responder: qual o conhecimento, a atitude e a prática de mulheres profissionais do sexo quanto à prevenção de infecções sexualmente transmissíveis, antes e após uma intervenção educativa?

O objetivo principal desta pesquisa desenvolvida como dissertação de mestrado foi avaliar o conhecimento, atitude e prática de mulheres profissionais do sexo sobre prevenção de infecções sexualmente transmissíveis, antes e após uma intervenção educativa.

\section{Metodologia}

Realizou-se um estudo descritivo, transversal, com abordagem quantitativa, do tipo estudo de intervenção não controlado, chamado antes e depois, nos quais as pessoas recebem a intervenção e sua condição é verificada antes do início e após (Escosteguy, 2009). A população do estudo foi composta por mulheres em situação de prostituição que atuavam em ambientes privados (casas de prostituição) na cidade de Sobral, município da região noroeste do Estado do Ceará, localizado a 240 km da capital Fortaleza. Para definição do grupo em estudo, elegeu-se os critérios de inclusão: ter idade igual ou superior a 18 anos e que estivessem em situação de prostituição em ambientes privados por um período igual ou superior a seis meses.

A coleta de dados foi realizada nos meses de abril e maio de 2016 por duas enfermeiras, sendo uma delas mestranda do Mestrado Profissional em Saúde da Família/RENASF. As três etapas (pré-teste, intervenção educativa e pós-teste) ocorreram em quatro casas de prostituição, em datas previamente agendadas conforme conveniência das participantes, com 75 mulheres profissionais do sexo.

Nestas ocasiões foram utilizados os seguintes instrumentos de coleta de dados: [i] roteiro de questionário sociodemográfico e do trabalho (12 e 8 questões, respectivamente); [ii] inquérito de Conhecimento, Atitude e Prática (inquérito CAP), com 17 questões sobre conhecimento, 22 questões sobre atitudes/crenças e 15 questões sobre a prática. A elaboração dos itens que compuseram o inquérito foi adaptado da pesquisa "Avaliação da efetividade das ações de prevenção dirigidas às profissionais do sexo, em três regiões brasileiras" desenvolvida pela Secretaria executiva do Programa Nacional de DST e 
AIDS, do Ministério da Saúde (2004) que subsidiou o inquérito CAP fundamentado nos documentos disponibilizados pelo ministério da saúde (BRASIL, 2015).

O Inquérito CAP foi aplicado antes da intervenção e imediatamente após, sendo atribuído o conceito "adequado" ou "inadequado" para cada dimensão do inquérito CAP, considerando os seguintes critérios:

\section{A) Conhecimento:}

- Adequado: Quando a mulher em situação de prostituição diz conhecer as principais DST e HIV; o método correto de prevenção em todos os tipos de práticas sexuais (anal, vaginal e oral) realizadas; a importância dos testes sorológicos rápidos para HIV, Sífilis e Hepatites; do exame preventivo ginecológico para a diagnóstico precoce das DST.

- Inadequado: Quando a trabalhadora do sexo diz não conhecer as principais DST e HIV, não saber o método correto para evitar as DST/HIV em todos os programas e nas diferentes práticas sexuais (anal, vaginal e oral) e o desconhecimento da importância dos testes sorológicos rápidos para HIV, Sífilis e Hepatites, do exame preventivo ginecológico para a diagnóstico precoce das DST.

B) Atitude:

- Adequada: Quando a mulher em situação de prostituição concorda totalmente em ser responsável na prevenção das DST/HIV com o uso do preservativo em todos os programas, e desistindo destes, caso não disponha do preservativo.

- Inadequado: Quando a mulher em situação de prostituição concorda em parte ou discorda que seja responsável pela prevenção das DST/HIV. Quando a mulher não recusa programas na ausência dos preservativos.

C) Prática:

- Adequada: Quando a mulher em situação de prostituição refere sempre fazer uso de preservativo em todos os programas, inclusive com o companheiro e reconhece as principais DST/HIV e realiza periodicamente os testes sorológicos rápidos para HIV, Súfilis, Hepatites, exame preventivo ginecológico para a diagnóstico precoce das DST.

- Inadequada: Quando a trabalhadora do sexo refere não usar ou usar somente algumas vezes o preservativo nos programas, como, também, quando não usar com o companheiro e não realiza periodicamente os testes sorológicos rápidos para HIV, Sífilis, Hepatites, exame preventivo ginecológico para a diagnóstico precoce das DST.

A intervenção educativa de Enfermagem intitulada "Promovendo o Empoderamento e a Saúde Sexual de Mulheres" com carga horaria de 20 horas foi realizada em 5 encontros de 4 horas, em dias alternados, nas quatro casas de prostituição, envolvendo uma média de 18 mulheres/encontro, sendo abordadas as seguintes temáticas: 1. Acolhimento afetivo e apresentação inicial do curso e da pesquisa; 2. Saúde da Mulher (Direitos Humanos, Políticas Públicas); 3.IST/HIV (O que é? como se pega? como se previne?) incorporando compreensão sobre "Risco" e "Vulnerabilidade; 4. Desenvolvendo a autoestima e autonomia das mulheres; 5. Conhecimento, Atitude e Prática e encerramento. Os encontros pedagógicos contemplaram dinâmicas de integração e acolhimento para fortalecimento e afetividade entre as mulheres e facilitadoras, com estratégias pedagógicas envolvendo: situações problema; vídeos de apoio; rodas de conversa; construção da árvore do 
problema (IST/HIV), dinâmicas para elevar a autoestima e desenvolver a autonomia para tomada de decisões alinhadas assertivas para promoção da saúde e avaliação ao final de cada encontro.

Por ocasião da intervenção educativa foram ofertados também testes rápidos para HIV, Hepatites e Sífilis, em parceria com o Centro de Orientação e Aconselhamento Sorológico - COAS/Secretaria de Saúde de Sobral e certificados de curso de extensão de curta duração pela participação no curso, emitidos pela Pró-reitoria de Extensão da Universidade Estadual Vale do Acaraú (PROEX-UVA).

Os dados foram digitados no Excel versão 12 e analisados no software StatisticalPackage for the Social Sciences (SPSS) versão 19.0. O teste de McNemar foi utilizado para comparação dos domínios do CAP antes e após a intervenção.

Este estudo obedeceu aos princípios éticos da Resolução 466/12 do Conselho Nacional de Saúde e foi submetido ao Comitê de Ética em Pesquisa da Universidade Estadual do Vale do Acaraú - UVA, sendo aprovado pelo parecer número: 1.450 .232 .

\section{Resultados}

Os resultados foram agrupados em quatro tópicos: Caracterização das mulheres em situação de prostituição em Sobral - CE; Conhecimentos de mulheres em situação de prostituição sobre prevenção de IST/HIV, antes e após a intervenção educativa; Atitudes de mulheres em situação de prostituição sobre prevenção de IST/HIV, antes e após a intervenção educativa; Práticas de mulheres profissionais do sexo sobre prevenção de IST/HIV, antes e após a intervenção educativa.

\subsection{Caracterização das mulheres em situação de prostituição do município de Sobral-CE}

Tabela 1. Distribuição das mulheres em situação de prostituição conforme os dados sociodemográficos e de condições de saúde. Sobral, junho de 2016.

\begin{tabular}{|c|c|c|}
\hline ACOMETIMENTO POR IST/HIV & $\mathbf{N}$ & $\%$ \\
\hline \multicolumn{3}{|l|}{$\begin{array}{l}\text { Teve alguma vez na vida uma doença } \\
\text { que se pega pelo sexo }\end{array}$} \\
\hline Sim & 26 & $34,7 \%$ \\
\hline Não & 49 & $65,3 \%$ \\
\hline Total & 75 & $100,0 \%$ \\
\hline \multicolumn{3}{|l|}{ Tipo de doença $(n=26)$} \\
\hline Gonorreia & 4 & $15,4 \%$ \\
\hline Herpes Genital & 5 & $19,2 \%$ \\
\hline HPV & 5 & $19,2 \%$ \\
\hline Sifilis & 12 & $46,2 \%$ \\
\hline \multicolumn{3}{|l|}{ Local de tratamento } \\
\hline Rede Pública & 24 & $92,3 \%$ \\
\hline Rede Privada & 2 & $7,7 \%$ \\
\hline Total & 26 & $100,0 \%$ \\
\hline Faixa Etária & $\mathbf{N}$ & $\%$ \\
\hline Até 20 anos & 10 & $13,3 \%$ \\
\hline 21 a 30 anos & 48 & $64,0 \%$ \\
\hline 31 a 40 anos & 15 & $20,0 \%$ \\
\hline$>40$ anos & 2 & $2,7 \%$ \\
\hline Total & 75 & $100,0 \%$ \\
\hline
\end{tabular}




\section{Estado Civil}

Solteira

Casada ou vive com alguém

Separada/divorciada

Viúva

Total

27

12

35

1

75

Escolaridade

Fundamental I incompleto (1a a 4a série)

Fundamental II incompleto ( 5 a a 8 a série)

Fundamental Completo

Ensino médio incompleto

Ensino médio completo

Total

VARIÁVEIS

Tempo de trabalho

\begin{tabular}{lcc}
$<1$ ano & 6 & $8,0 \%$ \\
\hline 1 a 2 anos & 27 & $36,0 \%$ \\
\hline 3 a 5 anos & 27 & $36,0 \%$ \\
\hline 5 a 10 anos & 14 & $18,7 \%$ \\
\hline$>10$ anos & 1 & $1,3 \%$ \\
\hline Total & 75 & $100,0 \%$
\end{tabular}

\section{Quantidade de programas por semana}

\begin{tabular}{lcc}
\hline 3 programas & 2 & $2,7 \%$ \\
\hline 5 Programas & 21 & $28,0 \%$ \\
\hline Mais de 5 programas & 52 & $69,3 \%$ \\
\hline Total & 75 & $100,0 \%$ \\
\hline
\end{tabular}

Fonte: Autores.

Das 75 (100\%) mulheres em situação de prostituição que participaram do estudo, parte significativa vive em uma situação de vida bastante desfavorável, considerando que uma alta proporção 27 (36,0\%) são solteiras, 35 (46,7\%) separadas/divorciadas, 37 (77,3\%) são jovens com média de idade de 26,7 anos, a maioria das mulheres 44 (58,7\%) mora com a família (pai, mãe, irmão e filhos), observou- se que 61 (81\%) tinham filhos e 50 (81,9\%) entre um e dois filhos. Em relação ao tempo de trabalho em situação de prostituição, foi notório o número de mulheres ingressando na prostituição recentemente, visto que $70(80,0 \%)$ exerciam essa prática de seis meses até cinco anos. A migração é outro fator presente no estudo, pois 35 $(46,7 \%)$ mulheres eram advindas de outras regiões e Estados.

Em relação ao número de programas realizados por semana, observamos predominância de 52 (69,3\%) mulheres, atendendo mais de cinco clientes por semana e cobrando baixo valor pelos programas e apresentando baixa renda mensal, pois constatamos que a maioria das mulheres 49 (65,3\%) ganhava entre 30-50 reais por programa, com uma média de 43,13 reais (DP: 15,81), e em relação a renda familiar mensal mostraram que 36 (48\%) das profissionais do sexo tem uma renda de 500 a 1000 reais, com uma média de 892,29. Quanto ao exercício de outra atividade paralela a prostituição, observamos que 71 $(94,7 \%)$ das participantes não exerciam outro trabalho vivendo apenas da prostituição, possuindo baixo nível de escolaridade, a maioria 63 (84\%) não tinham concluído 10 anos de estudos. 
O relato de acometimento de IST nas participantes do estudo foi de 26 casos $(34,7 \%)$. Isoladamente, Sífilis foi a IST mais frequente $12(45,1 \%)$, seguida respectivamente de HPV, $5(19,2 \%)$; Herpes Genital, 5 (19,2\%) e Gonorreia, 4 (15,4\%). Sem nenhum relato de existência para HIV/Aids, e a maioria, 24 (92,3\%) tratadas na rede pública pelo SUS.

\subsection{Conhecimentos de mulheres em situação de prostituição sobre a prevenção das IST/HIV}

Tabela 2. Avaliação da adequação e inadequação do conhecimento das mulheres em situação de prostituição sobre a prevenção das IST/HIV, antes e após a intervenção. Sobral-CE, junho de 2016.

\begin{tabular}{|c|c|c|c|c|c|}
\hline \multirow{2}{*}{ CONHECIMENTO } & \multicolumn{2}{|c|}{ Pré-teste } & \multicolumn{2}{|c|}{ Pós-teste } & \multirow{2}{*}{$\mathbf{P}$} \\
\hline & $\mathbf{N}$ & $\%$ & $\mathbf{N}$ & $\%$ & \\
\hline \multicolumn{6}{|l|}{$\begin{array}{l}\text { 1.Tipos de prática sexual que pode ser } \\
\text { utilizado camisinha na prevenção das DST } \\
\text { e AIDS }\end{array}$} \\
\hline Sexo oral & & & & & $<0,01$ \\
\hline Sim & 62 & $82,7 \%$ & 74 & $98,7 \%$ & \\
\hline Não & 13 & $17,3 \%$ & 1 & $1,3 \%$ & \\
\hline Sexo vaginal & & & & & $*$ \\
\hline Sim & 75 & $100,0 \%$ & 75 & $100,0 \%$ & \\
\hline Não & 0 & $0,0 \%$ & 0 & $0,0 \%$ & \\
\hline Sexo anal & & & & & $* *$ \\
\hline Sim & 73 & $97,3 \%$ & 75 & $100,0 \%$ & \\
\hline Não & 2 & $2,7 \%$ & 0 & $0,0 \%$ & \\
\hline INADEQUADO & 15 & $20,0 \%$ & 1 & $1,3 \%$ & \\
\hline ADEQUADO & 60 & $80,0 \%$ & 74 & $98,7 \%$ & \\
\hline \multicolumn{6}{|l|}{ 2. Métodos que evitam as DST e HIV } \\
\hline Camisinha & & & & & $*$ \\
\hline Sim & 75 & $100,0 \%$ & 75 & $100,0 \%$ & \\
\hline Não & 0 & $0,0 \%$ & 0 & $0,0 \%$ & \\
\hline Pílula & & & & & $* *$ \\
\hline Sim & 7 & $9,3 \%$ & 0 & $0,0 \%$ & \\
\hline Não & 68 & $90,7 \%$ & 75 & $100,0 \%$ & \\
\hline Coito interrompido & & & & & $* *$ \\
\hline Sim & 5 & $6,7 \%$ & 0 & $0,0 \%$ & \\
\hline Não & 70 & $93,3 \%$ & 75 & $100,0 \%$ & \\
\hline Injeção & & & & & $* *$ \\
\hline Sim & 2 & $2,7 \%$ & 0 & $0,0 \%$ & \\
\hline Não & 73 & $97,3 \%$ & 75 & $100,0 \%$ & \\
\hline Preservativo feminino & & & & & $<0,01$ \\
\hline Sim & 21 & $28,0 \%$ & 65 & $86,7 \%$ & \\
\hline Não & 54 & $72,0 \%$ & 10 & $13,3 \%$ & \\
\hline INADEQUADO & 68 & $90,7 \%$ & 10 & $13,3 \%$ & \\
\hline ADEQUADO & 7 & $9,4 \%$ & 65 & $86,7 \%$ & \\
\hline $\begin{array}{l}\text { 3. A não utilização de preservativo com } \\
\text { parceiro conhecido evita DST/HIV }\end{array}$ & & & & & 0,39 \\
\hline Sim & 10 & $13,3 \%$ & 6 & $8,0 \%$ & \\
\hline Não & 65 & $86,7 \%$ & 69 & $92,0 \%$ & \\
\hline INADEQUADO & 10 & $13,3 \%$ & 6 & $8,0 \%$ & \\
\hline ADEQUADO & 65 & $86,7 \%$ & 69 & $92,0 \%$ & \\
\hline \multicolumn{6}{|l|}{$\begin{array}{l}\text { 4. Infecções transmitidas por meio do ato } \\
\text { sexual }\end{array}$} \\
\hline Sífilis & & & & & $* *$ \\
\hline Sim & 69 & $92,0 \%$ & 75 & $100,0 \%$ & \\
\hline Não & 6 & $8,0 \%$ & 0 & $0,0 \%$ & \\
\hline
\end{tabular}




\begin{tabular}{|c|c|c|c|c|c|}
\hline \multirow{2}{*}{ CONHECIMENTO } & \multicolumn{2}{|c|}{ Pré-teste } & \multicolumn{2}{|c|}{ Pós-teste } & \multirow{2}{*}{$\mathbf{P}$} \\
\hline & $\mathbf{N}$ & $\%$ & $\mathbf{N}$ & $\%$ & \\
\hline INADEQUADO & 6 & $92,0 \%$ & 75 & $100,0 \%$ & \\
\hline ADEQUADO & 69 & $8,0 \%$ & 0 & $0,0 \%$ & \\
\hline Aids/HIV & & & & & * \\
\hline Sim & 75 & $100,0 \%$ & 75 & $100,0 \%$ & \\
\hline Não & 0 & $0,0 \%$ & 0 & $0,0 \%$ & \\
\hline INADEQUADO & 0 & $0,0 \%$ & 0 & $0,0 \%$ & \\
\hline ADEQUADO & 75 & $100,0 \%$ & 75 & $100,0 \%$ & \\
\hline Hepatites & & & & & $<0,01$ \\
\hline Sim & 31 & $41,3 \%$ & 72 & $96,0 \%$ & \\
\hline Não & 41 & $54,7 \%$ & 3 & $4,0 \%$ & \\
\hline INADEQUADO & 41 & $54,7 \%$ & 3 & $96,0 \%$ & \\
\hline ADEQUADO & 31 & $41,3 \%$ & 72 & $4,0 \%$ & \\
\hline Gonorreia & & & & & $<0,01$ \\
\hline Sim & 46 & $61,3 \%$ & 70 & $93,3 \%$ & \\
\hline Não & 29 & $38,7 \%$ & 5 & $6,7 \%$ & \\
\hline INADEQUADO & 29 & $38,7 \%$ & 5 & $6,7 \%$ & \\
\hline ADEQUADO & 46 & $61,3 \%$ & 70 & $93,3 \%$ & \\
\hline Clamídia & & & & & $<0,01$ \\
\hline Sim & 21 & $28,0 \%$ & 70 & $93,3 \%$ & \\
\hline Não & 54 & $72,0 \%$ & 5 & $6,7 \%$ & \\
\hline INADEQUADO & 54 & $72,0 \%$ & 5 & $6,7 \%$ & \\
\hline ADEQUADO & 21 & $28,0 \%$ & 70 & $93,3 \%$ & \\
\hline Herpes & & & & & $<0,01$ \\
\hline Sim & 40 & $53,3 \%$ & 74 & $98,7 \%$ & \\
\hline Não & 35 & $46,7 \%$ & 1 & $1,3 \%$ & \\
\hline INADEQUADO & 35 & $46,7 \%$ & 1 & $1,3 \%$ & \\
\hline ADEQUADO & 40 & $53,3 \%$ & 70 & $98,7 \%$ & \\
\hline $\begin{array}{l}\text { 5. Autopercepção do risco de pegar DST/ } \\
\text { HIV }\end{array}$ & & & & & **** \\
\hline É Grande & 37 & $49,3 \%$ & 72 & $96,0 \%$ & \\
\hline É mais ou menos (médio) & 17 & $22,7 \%$ & 0 & $0,0 \%$ & \\
\hline É Pequeno & 8 & $10,7 \%$ & 0 & $0,0 \%$ & \\
\hline Nenhum & 3 & $4,0 \%$ & 2 & $2,7 \%$ & \\
\hline Não sabe avaliar & 10 & $13,3 \%$ & 1 & $1,3 \%$ & \\
\hline INADEQUADO & 28 & $50,7 \%$ & 3 & $4,0 \%$ & \\
\hline ADEQUADO & 37 & $49,3 \%$ & 72 & $96,0 \%$ & \\
\hline
\end{tabular}

Fonte: Autores.

Em relação ao conhecimento, sobre a prevenção das IST/HIV antes da intervenção 60 (80\%) reconheceram ser necessário o uso nas três práticas sexuais, após a intervenção esse percentual ascendeu para 74 (98,7\%).

Sobre os vários métodos contraceptivos citados, foi questionado qual seriam os adequados para a prevenção das IST/HIV, sendo as respostas corretas, os preservativos femininos e masculinos, antes da intervenção todas as mulheres 75 (100\%) afirmaram que o preservativo masculino é um método correto, em contrapartida, 54 (72\%) profissionais do sexo responderam que o preservativo feminino não protege contra as IST/HIV. Após a intervenção educativa apenas 10 (13,3\%) mulheres continuaram afirmando que o preservativo feminino não protege contra as IST/HIV. Abordar os diversos tipos de prática sexual, destacando as diferentes vulnerabilidades masculinas e femininas (biológica e de gênero) é fundamental para que homens e mulheres percebam as situações de risco que vivenciam, não apenas a partir do seu comportamento sexual, mas também de suas parcerias (Nicolau et al., 2016). 
No tocante ao conhecimento das mulheres sobre as infecções transmitidas através do sexo, as mais citadas foram: HIV/Aids $75(100 \%)$, Sífilis $69(92 \%)$ e gonorreia $46(61,3 \%)$, contudo um percentual significativo afirmaram não conhecer as seguintes IST: clamídia 54 (72\%), hepatites 41 (54,7\%) e herpes 35 (46,7\%), após a intervenção houve melhora com o aumento dos percentuais para Súfilis 75 (100\%), hepatites 72 (96\%), gonorreia 70 (93,3\%), clamídia 70 (93,3\%) e herpes 74 $(98,7 \%)$.

No que concerne a autopercepção de risco em adquirir uma IST/HIV, antes da intervenção parte significativa 17 $(22,7 \%)$ afirmaram ter médio risco para adquirir, $8(10,7 \%)$ pequeno, $13(17,3 \%)$ nenhum/não sabe avaliar e menos da metade 37 (49,3\%) afirmaram ser grande o risco para a aquisição de DST/HIV; após a intervenção, o estudo revelou melhoria na autopercepção do risco quando $72(96 \%)$ das profissionais do sexo afirmaram ter um grande risco.

\subsection{Atitudes das mulheres em situação de prostituição sobre a prevenção das IST/HIV}

Tabela 3. Avaliação da adequação e inadequação das atitudes e crenças de mulheres em situação de prostituição sobre a prevenção das IST/HIV, antes e após a intervenção. Sobral-CE, junho de 2016.

\begin{tabular}{|c|c|c|c|c|c|}
\hline \multirow{2}{*}{ ATITUDE } & \multicolumn{2}{|c|}{ Pré-teste } & \multicolumn{2}{|c|}{ Pós-teste } & \multirow{2}{*}{$\mathbf{P}$} \\
\hline & $\mathbf{N}$ & $\%$ & $\mathbf{N}$ & $\%$ & \\
\hline $\begin{array}{l}\text { 1. Importância de ter preservativo em } \\
\text { todo momento }\end{array}$ & & & & & $* *$ \\
\hline Sim & 73 & $97,3 \%$ & 75 & $100,0 \%$ & \\
\hline Não & 2 & $2,7 \%$ & 0 & $0,0 \%$ & \\
\hline INADEQUADO & 2 & $2,7 \%$ & 0 & $0,0 \%$ & \\
\hline ADEQUADO & 73 & $97,3 \%$ & 75 & $100,0 \%$ & \\
\hline $\begin{array}{l}\text { 2. Atitude sobre um programa sem } \\
\text { preservativo }\end{array}$ & & & & & $* * *$ \\
\hline Desiste do programa & 42 & $56,0 \%$ & 50 & $66,7 \%$ & \\
\hline $\begin{array}{l}\text { Dá um jeito de comprar (você ou o } \\
\text { cliente) }\end{array}$ & 12 & $16,0 \%$ & 25 & $33,3 \%$ & \\
\hline $\begin{array}{l}\text { Faz o programa, por um preço mais } \\
\text { alto }\end{array}$ & 21 & $28,0 \%$ & 0 & $0,0 \%$ & \\
\hline INADEQUADO & 21 & $28,0 \%$ & 0 & $0,0 \%$ & \\
\hline ADEQUADO & 54 & $72,0 \%$ & 75 & $100,0 \%$ & \\
\hline & $\mathrm{N}$ & $\%$ & $\mathrm{~N}$ & $\%$ & \\
\hline $\begin{array}{l}\text { 3. Você acredita que uma pessoa pode } \\
\text { pegar DST/HIV de alguma dessas } \\
\text { maneiras }\end{array}$ & & & & & \\
\hline Através de toalhas e lençóis & & & & & $* * *$ \\
\hline Sim & 35 & $47,9 \%$ & 10 & $13,3 \%$ & \\
\hline Não & 35 & $47,9 \%$ & 65 & $86,7 \%$ & \\
\hline Não sabe & 3 & $4,1 \%$ & 0 & $0,0 \%$ & \\
\hline INADEQUADO & 38 & $52,0 \%$ & 10 & $13,3 \%$ & \\
\hline ADEQUADO & 35 & $47,9 \%$ & 65 & $86,7 \%$ & \\
\hline $\begin{array}{l}\text { 4. Comendo no mesmo prato ou } \\
\text { bebendo no mesmo copo }\end{array}$ & & & & & $* * *$ \\
\hline Sim & 39 & $52,0 \%$ & 11 & $14,7 \%$ & \\
\hline Não & 33 & $44,0 \%$ & 64 & $85,3 \%$ & \\
\hline Não sabe & 3 & $4,0 \%$ & 0 & $0,0 \%$ & \\
\hline INADEQUADO & 42 & $56,0 \%$ & 11 & $14,7 \%$ & \\
\hline ADEQUADO & 33 & $44,0 \%$ & 64 & $85,3 \%$ & \\
\hline
\end{tabular}




\begin{tabular}{|c|c|c|c|c|c|}
\hline \multirow{2}{*}{ ATITUDE } & \multicolumn{2}{|c|}{ Pré-teste } & \multicolumn{2}{|c|}{ Pós-teste } & \multirow{2}{*}{$\mathbf{P}$} \\
\hline & $\mathbf{N}$ & $\%$ & $\mathbf{N}$ & $\%$ & \\
\hline Sim & 74 & $98,7 \%$ & 74 & $98,7 \%$ & \\
\hline Não & 0 & $0,0 \%$ & 1 & $1,3 \%$ & \\
\hline Não sabe & 1 & $1,3 \%$ & 0 & $0,0 \%$ & \\
\hline INADEQUADO & 1 & $1,3 \%$ & 1 & $1,3 \%$ & \\
\hline ADEQUADO & 74 & $98,7 \%$ & 74 & $98,7 \%$ & \\
\hline 6. Sexo anal sem preservativo & & & & & * \\
\hline Sim & 75 & $100,0 \%$ & 75 & $100,0 \%$ & \\
\hline Não & 0 & $0,0 \%$ & 0 & $0,0 \%$ & \\
\hline INADEQUADO & 0 & $0,0 \%$ & 0 & $0,0 \%$ & \\
\hline ADEQUADO & 75 & $100,0 \%$ & 75 & $100,0 \%$ & \\
\hline 7. Sexo vaginal sem preservativo & & & & & $*$ \\
\hline Sim & 75 & $100,0 \%$ & 75 & $100,0 \%$ & \\
\hline Não & 0 & $0,0 \%$ & 0 & $0,0 \%$ & \\
\hline INADEQUADO & 0 & $0,0 \%$ & 0 & $0,0 \%$ & \\
\hline ADEQUADO & 75 & $100,0 \%$ & 75 & $100,0 \%$ & \\
\hline 8. Sexo oral sem preservativo & & & & & ** \\
\hline Sim & 69 & $92,0 \%$ & 75 & $100,0 \%$ & \\
\hline Não & 1 & $1,3 \%$ & 0 & $0,0 \%$ & \\
\hline Não sabe & 5 & $6,7 \%$ & 0 & $0,0 \%$ & \\
\hline INADEQUADO & 6 & $8,0 \%$ & 0 & $0,0 \%$ & \\
\hline ADEQUADO & 69 & $92,0 \%$ & 75 & $100,0 \%$ & \\
\hline 9. Pela picada de insetos & & & & & $* * *$ \\
\hline Sim & 43 & $57,3 \%$ & 13 & $17,3 \%$ & \\
\hline Não & 27 & $36,0 \%$ & 62 & $82,7 \%$ & \\
\hline Não sabe & 5 & $6,7 \%$ & 0 & $0,0 \%$ & \\
\hline INADEQUADO & 48 & $64,0 \%$ & 13 & $17,3 \%$ & \\
\hline ADEQUADO & 27 & $36,0 \%$ & 62 & $82,7 \%$ & \\
\hline $\begin{array}{l}\text { 10. Durante a gravidez ou o parto (da } \\
\text { mãe para o filho) }\end{array}$ & & & & & $* * *$ \\
\hline Sim & 52 & $69,3 \%$ & 67 & $91,8 \%$ & \\
\hline Não & 9 & $12,0 \%$ & 6 & $8,2 \%$ & \\
\hline Não sabe & 14 & $18,7 \%$ & 0 & $0,0 \%$ & \\
\hline INADEQUADO & 23 & $20,7 \%$ & 6 & $8,2 \%$ & \\
\hline ADEQUADO & 52 & $69,3 \%$ & 62 & $91,8 \%$ & \\
\hline 11. Pela amamentação & & & & & $* * *$ \\
\hline Sim & 31 & $41,3 \%$ & 70 & $93,3 \%$ & \\
\hline Não & 23 & $30,7 \%$ & 5 & $6,7 \%$ & \\
\hline Não sabe & 21 & $28,0 \%$ & 0 & $0,0 \%$ & \\
\hline INADEQUADO & 44 & $58,7 \%$ & 5 & $6,7 \%$ & \\
\hline ADEQUADO & 31 & $41,3 \%$ & 70 & $93,3 \%$ & \\
\hline 12. Pelo uso de vaso sanitário & & & & & $* * *$ \\
\hline Sim & 51 & $68,0 \%$ & 12 & $16,0 \%$ & \\
\hline Não & 22 & $29,3 \%$ & 63 & $84,0 \%$ & \\
\hline Não sabe & 14 & $18,7 \%$ & 0 & $0,0 \%$ & \\
\hline INADEQUADO & 65 & $86,7 \%$ & 6 & $8,2 \%$ & \\
\hline ADEQUADO & 22 & $29,3 \%$ & 63 & $84,0 \%$ & \\
\hline
\end{tabular}




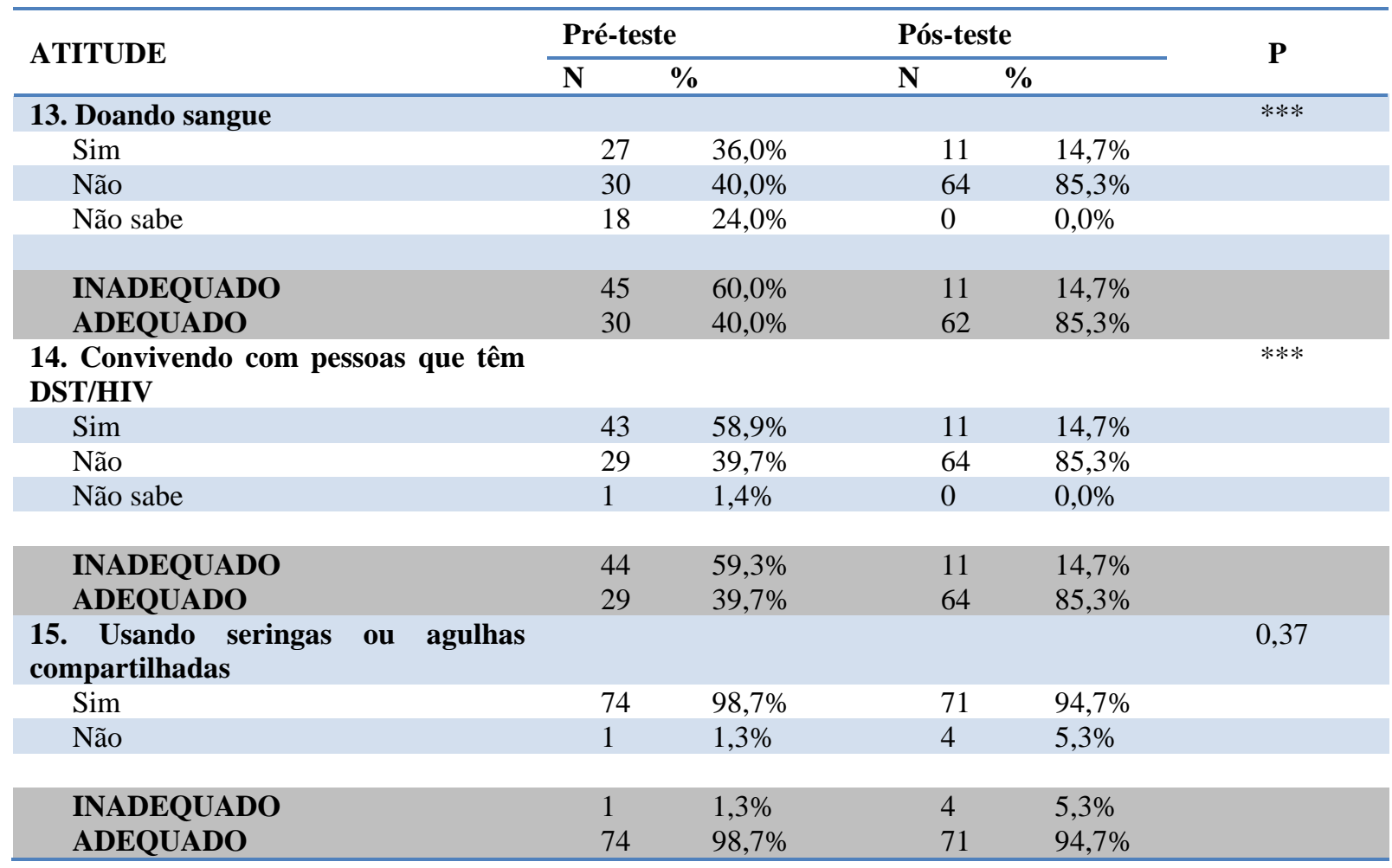

Fonte: Autores.

Foi alto o número de mulheres em situação de prostituição que consideram uma atitude importante ter com elas preservativos em todo momento, já antes da intervenção educativa, pois houve predomínio da afirmação positiva (sim), tanto antes como após a intervenção $73(97,3 \%)$ e 75 (100,0\%) respectivamente.

Em relação à realização de programas sem preservativos, antes da intervenção um percentual significativo 21 (28\%) respondeu aceitar realizar o programa por um preço mais alto, após a intervenção todas as mulheres 75 (100\%) afirmaram que desistiriam do programa ou dariam um jeito de comprar o preservativo.

Em relação aos meios que realmente constituem-se formas de transmissão das IST/HIV. Observa-se que as participantes reconhecem os meios corretos mesmo antes da intervenção educativa quando identificam que o sexo anal, vaginal e oral sem preservativo transmitem IST/HIV com 75 (100\%), $75(100 \%)$ e 69 (92\%) respectivamente.

Também foi alta a proporção de participantes que identificam a transmissão de IST/HIV durante a gravidez e parto 52 $(69,3 \%)$. A amamentação foi o meio de transmissão correto menos identificado pelas participantes 31 (41,3\%) antes da intervenção e, após a intervenção esse percentil ascendeu para 70 (93,3\%).

Em relação aos meios que não se constituem forma de transmissão das IST/HIV, os dados revelam muita desinformação antes da intervenção educativa. com a identificação positivados meios: recebendo transfusão de sangue 46 $(61,3 \%)$, pelo uso do vaso sanitário $51(68 \%)$ e pela picada de insetos por $43(57,3 \%)$ e, após a intervenção houve melhora significativa na atitude dessas mulheres com a diminuição da desinformação sobre os meios para: recebendo transfusão de sangue 15 (20\%), pelo uso do vaso sanitário 12 (16\%) e pela picada de insetos $13(17,3 \%)$. 


\subsection{Prática das mulheres em situação de prostituição sobre a prevenção das IST/HIV}

Tabela 4. Avaliação da adequação e inadequação das práticas de mulheres em situação de prostituição sobre a prevenção das IST/HIV, antes e após a intervenção. Sobral-CE, junho de 2016.

\begin{tabular}{|c|c|c|c|c|c|}
\hline \multirow{2}{*}{ PRÁTICA - IST/HIV } & \multicolumn{2}{|c|}{ Pré-teste } & \multicolumn{2}{|c|}{ Pós-teste } & \multirow{2}{*}{$\mathbf{P}$} \\
\hline & $\mathbf{N}$ & $\%$ & $\mathbf{N}$ & $\%$ & \\
\hline \multicolumn{6}{|l|}{ 1. Com quem não utiliza a camisinha } \\
\hline Com cliente preferencial & 29 & $38,7 \%$ & 29 & $38,7 \%$ & $*$ \\
\hline Com marido / companheiro & 38 & $50,7 \%$ & 38 & $50,7 \%$ & $*$ \\
\hline Com amigo & 0 & $0,0 \%$ & 0 & $0,0 \%$ & $*$ \\
\hline Com cliente que recusa & 0 & $0,0 \%$ & 0 & $0,0 \%$ & $*$ \\
\hline Com cliente que paga mais & 15 & $20,0 \%$ & 15 & $20,0 \%$ & $*$ \\
\hline Com cliente que ameaça & 0 & $0,0 \%$ & 0 & $0,0 \%$ & $*$ \\
\hline Com mulheres & 1 & $1,3 \%$ & 1 & $1,3 \%$ & $*$ \\
\hline \multicolumn{6}{|l|}{ 2. Nos últimos seis meses, usou camisinha } \\
\hline Em todos os programas & 40 & $53,3 \%$ & 40 & $53,3 \%$ & $*$ \\
\hline Em menos da metade das vezes & 20 & $26,7 \%$ & 20 & $26,7 \%$ & \\
\hline Não respondeu/não sabe & 15 & $20,0 \%$ & 15 & $20,0 \%$ & \\
\hline \multicolumn{6}{|l|}{ 3. Motivos para deixar de usar a camisinha } \\
\hline É muito cara & 0 & $0,0 \%$ & 0 & $0,0 \%$ & * \\
\hline Não tem para comprar/é difícil de achar & 0 & $0,0 \%$ & 0 & $0,0 \%$ & $*$ \\
\hline Não gosta de usar & 0 & $0,0 \%$ & 0 & $0,0 \%$ & $*$ \\
\hline Tira o prazer/quebra o clima & 0 & $0,0 \%$ & 0 & $0,0 \%$ & $*$ \\
\hline O parceiro/cliente não gosta/ se recusa & 5 & $6,7 \%$ & 5 & $6,7 \%$ & $*$ \\
\hline Não precisa, confia no parceiro & 39 & $52,0 \%$ & 39 & $52,0 \%$ & $*$ \\
\hline Perde o cliente & 36 & $48,0 \%$ & 36 & $48,0 \%$ & * \\
\hline Não sabe usar & 0 & $0,0 \%$ & 0 & $0,0 \%$ & $*$ \\
\hline $\begin{array}{l}\text { Tem vergonha de pedir para o parceiro ou } \\
\text { o cliente }\end{array}$ & 0 & $0,0 \%$ & 0 & $0,0 \%$ & $*$ \\
\hline Não acredita que protege contra a AIDS & 0 & $0,0 \%$ & 0 & $0,0 \%$ & $*$ \\
\hline $\begin{array}{l}\text { Tem medo de usar/acha que causa } \\
\text { problemas de saúde }\end{array}$ & 0 & $0,0 \%$ & 0 & $0,0 \%$ & $*$ \\
\hline \multicolumn{6}{|l|}{ 4. Forma de obter a camisinha } \\
\hline Compra & 3 & $4,0 \%$ & 3 & $4,0 \%$ & $*$ \\
\hline Recebe no local de trabalho & 73 & $97,3 \%$ & 73 & $97,3 \%$ & * \\
\hline Nunca tem camisinha & 0 & $0,0 \%$ & 0 & $0,0 \%$ & $*$ \\
\hline Serviço de saúde & 14 & $18,7 \%$ & 14 & $18,7 \%$ & * \\
\hline $\mathrm{Na} \mathrm{ONG}$ & 2 & $2,7 \%$ & 2 & $2,7 \%$ & $*$ \\
\hline $\begin{array}{l}\text { Astra (Associação das Trabalhadoras do } \\
\text { sexo) }\end{array}$ & 1 & $1,3 \%$ & 1 & $1,3 \%$ & * \\
\hline
\end{tabular}

Fonte: Autores.

Dados alarmantes foram verificados sobre a prática do uso de preservativos nos programas realizados pelas mulheres em situação de prostituição, 29 (38,7\%) afirmaram não usar preservativos com cliente preferencial, 15 (20\%) com o cliente que paga mais e $38(50,7 \%)$ com marido ou companheiro. Ao serem indagadas sobre a frequência do uso dos preservativos nos últimos seis meses 40 (53,3\%) afirmaram utilizar em todos os programas, mas um percentual expressivo de 35 (46,7\%) não utilizou em menos da metade dos programas ou não souberam responder.

As participantes também foram questionadas sobre os motivos que as fazem não usar preservativo, 41 (54,7\%) deixa de usar porque o cliente não gosta ou para não perder o cliente e $39(52 \%)$ responderam não precisar, pois confiam no marido ou companheiro. Também foi evidenciado que não deixam de usar por falta do preservativo no ambiente de trabalho já que 73 $(97,3 \%)$ afirmaram receber preservativos no local de trabalho. 


\section{Discussão}

As mulheres jovens ingressam na prostituição principalmente devido à situação socioeconômica precária de suas famílias, caracterizando sua exclusão na sociedade (escola, consumo, mercado de trabalho, saúde e cultura) agravada pela situação de violência sexual e/ou domésticas sofridas (Nunes \& Andrade, 2009).

A população em estudo constituiu-se majoritariamente por mulheres jovens de idade entre 20 e 30 anos, ou seja, em plena fase reprodutiva, na qual estão mais expostas as complicações das infecções transmissíveis pelo sexo. Característica compreensível, pois a beleza e a juventude são requisitos para a comercialização do prazer sexual (Neri et al., 2013). Este fato está em concordância com estudos realizados nacionais e internacionais (Moura et al., 2010; Salmeron \& Pessoa, 2012; Matos et al., 2013; Martins, 2015). Sabe-se que a jovem entra na prostituição com vistas a melhores rendimentos e para a maior aquisição de bens de consumo, e condições que permitam uma melhor sobrevivência (Aquino et al., 2010).

O presente estudo apresentou uma predominância de mulheres solteiras e separadas. Este achado corrobora com pesquisa descritiva desenvolvida na zona Sul da cidade de São Paulo - SP, em que, das 50 profissionais do sexo, 38 (76\%) eram solteiras (Salmeron \& Pessoa, 2012).

A conciliação da vida profissional com a familiar é um dos grandes desafios enfrentados pelas profissionais do sexo. Estudo realizado com mulheres em situação de prostituição cambojanas lista diversos sentimentos emocionais e afetivos negativos relatados, como elevados níveis de estresse emocional em seus relacionamentos românticos, dificuldades em manter relacionamentos duradouros e sentimentos de solidão; afirmam que seus parceiros não contribuem significativamente para atenuar suas angústias e deixam de proporcionar-lhes conforto e apoio em resposta à estigmatização em relação à profissão (Todd et al., 2010).

O baixo nível de escolaridade apresentado no estudo, com 63 (84\%) mulheres em situação de prostituição que não tinham concluído 10 anos de estudos, corroboram com outros estudos (Todd et al., 2010), podendo ter relação com o exercício da prostituição ainda muito jovem, uma vez que ao ingressarem no mercado de trabalho surgem determinadas dificuldades, como desmotivação ou cansaço físico, acesso à escola, a sobrecarga e horários de trabalho levando ao abandono precoce dos estudos , contribuindo para a evasão escolar (Santos et al., 2009).

As dificuldades financeiras ou a pobreza absoluta, o baixo nível de escolaridade, compõem os obstáculos para a integração das mulheres em situação de prostituição no mercado de trabalho. Para aquelas que pertencem às classes mais baixas, as perspectivas de mudança de atividade ainda são menos viáveis em virtude da baixa escolaridade e da ausência de qualificação profissional (Moura et al., 2010).

A migração também está presente no estudo. Os descolamentos em razão do estigma vivenciado pelas mulheres em situação de prostituição e as perspectivas de melhoria das condições de vida, é notório quando verificamos os dados da migração de suas cidades natais, como o foi neste estudo. Esse resultado está em concordância com pesquisas realizadas que afirmam ser comum a migração de mulheres para outras localidades em busca de melhores condições de trabalho, sendo consideradas "novas" no mercado (Vieira, 2015). Observou-se no estudo que as mulheres buscam evitar o desgaste de sua imagem, despertando assim a expectativa de retorno no cliente.

Com relação à baixa renda familiar mensal, o Instituto Brasileiro de Geografia e Estatística (2015) relata que quase um terço da população, ou seja, 68 milhões de pessoas vivem com até meio salário mínimo per capita. A renda per capita média do brasileiro em 2015 foi 1,113 reais, porém a renda per capita do Ceará foi 680 reais. Ao acrescentar as pessoas que vivem sem rendimento, estima-se que 54 milhões possam ser consideradas pobres. As disparidades por regiões também existem: no Nordeste: quase $51 \%$ das pessoas vivem com menos de um salário mínimo, enquanto no Sudeste esse número é inferior a $18 \%$. Ou seja, percebemos que as participantes, especificamente, não estão condicionadas a baixa renda, visto que essa condição é predominante no País (Instituto Brasileiro de Geografia e Estatística, 2015). 
O relato de existência geral de IST obtida entre as mulheres em situação de prostituição participantes do estudo foi: 26 $(34,7 \%)$, em que relataram que já tiveram ou têm uma IST. Mas deve-se analisar que a baixa prevalência pode estar associada ao não conhecimento dos sintomas característicos. O diagnóstico de uma DST, muitas vezes, não é realizado, pois nem sempre as mulheres associam os sintomas a uma infecção de transmissão sexual, o que aumenta os casos de subdiagnóstico (Neri et al., 2013).

Este também é um grave problema de saúde pública, pois essas mulheres e provavelmente, seus parceiros/clientes, continuam disseminando doenças com outros prováveis parceiros. Portanto, comprova-se que o número de mulheres infectadas ainda é grande. A busca de afetividade nos relacionamentos, seja com o companheiro, namorado ou cliente fixo surge como possível fator impeditivo para adoção de comportamentos preventivos consistentes e seguros (Moura et al., 2010).

Ressalta-se que é possível estas mulheres estarem com um nível de conhecimento elevado acerca da importância do uso do preservativo para a prevenção das IST/HIV/Aids, quando $74(98,7 \%)$ reconheceram a necessidade de preservativo nas três práticas sexuais (anal, vaginal e oral).

Mesmo assim, reitera-se a importância da utilização de preservativo nas relações sexuais, pois se sabe que este é um método de prevenção contra a aquisição de DST/HIV. Desse modo, no México, foram entrevistadas 2401 mulheres em situação de prostituição sendo identificado que, entre aquelas que usavam preservativo com os clientes, a incidência do HIV foi 15 vezes menor que entre as que não o fizeram (Moura et al., 2010). Com isso, reporta-se à necessidade de atividades educativas com este grupo de mulheres, a fim de promover a saúde sexual delas, prevenindo-as da aquisição de IST/HIV.

Mesmo com toda a divulgação existente, verifica-se conhecimento superficial por parte das mulheres em situação de prostituição em relação as DST. As respostas revelaram que as mulheres em situação de prostituição conhecem algumas DST em geral, mas não têm conhecimento sobre as complicações de cada uma, especificamente. Diante dessa realidade, faz-se necessário que essas mulheres ampliem de modo detalhado seus conhecimentos sobre as DST, já que o sexo faz parte do seu cotidiano, tornando-as universalmente mais vulneráveis que as outras pessoas (Neri et al., 2013).

Uma informação importante mostrada no estudo entre as participantes foi o desconhecimento sobre o preservativo feminino. É importante que seja ampliada as opções de prevenção, de tal forma que os direitos às práticas sexuais sejam um direito de todos (Brasil, 2005). Entende-se que as mulheres anseiam por métodos que, ao mesmo tempo em que lhes garantem proteção, facilitem a negociação com seus parceiros, de tal modo que possam garantir relações sexuais protegidas.

Um estudo CAP com 35 mulheres sobre o uso de preservativo feminino realizado em Fortaleza, CE, evidenciou que após a educação em saúde, 85,3\% apresentaram conhecimento adequado, 62,9\% atitude moderada e 55,9\% prática substancial. As pesquisadoras concluíram que as mulheres estavam dispostas a utilizar o preservativo feminino, desde que houvesse intervenção educativa eficaz (Fernandes et al., 2012).

Em relação à autopercepção do risco de se infectarem por DST/HIV, as mulheres em situação de prostituição ao negarem o risco de se infectarem por DST/HIV exercendo a profissão podem deixar de adotar práticas sexuais seguras, principalmente com clientes, em menor poder de negociação do uso do preservativo, acima citados, ou com os parceiros com os quais essas mulheres mantém alguma relação de afetividade (Cunha, Moreira \& Lôbo, 2012).

Foi percebido que as mulheres em situação de prostituição sabem da necessidade de manter relações sexuais protegidas, mas se deixam levar pelo desejo de seus parceiros sexuais e pela falta de poder de negociação para utilização do preservativo. As mulheres em situação de prostituição recebem propostas que, para elas, são irrecusáveis. Clientes oferecem mais dinheiro para que os "programas" sejam realizados sem o uso de preservativos e estas, muitas vezes, aceitam praticar sexo desprotegido. Assim, tornam-se cada vez mais vulneráveis às DST/HIV. Muitas vezes essa maior "vulnerabilidade" ocorre devido às necessidades financeiras, que as obrigam a ter relações sexuais desprotegidas, ou por questões relacionadas ao uso do álcool e/ou drogas pelas mulheres em situação de prostituição ou seus clientes, ou os dois, e pelos preconceitos 
existentes contra essa população, por parte, tanto da sociedade, como dos clientes, contribuindo para uma baixa autoestima (Moura et al., 2010).

Persiste ainda entre as mulheres em situação de prostituição muitos mitos quanto à transmissão de IST/HIV, remanescentes da década de 80 com o início da epidemia do HIV, acreditando que com o compartilhamento de talheres com indivíduos infectados, uso de vaso sanitário e por meio de picada de mosquito podia se adquirir uma IST/HIV. Além disso, mais da metade das mulheres em situação de prostituição 44 (58,7\%) não reconhecem a transmissão durante a amamentação e um terço 23 (30,7\%) não sabem a respeito da transmissão vertical do HIV/IST. Dado considerado um importante problema de saúde pública, visto que a população em estudo está em plena atividade reprodutiva e possui vários parceiros sexuais, inclusive sem o uso do preservativo.

As mulheres em situação de prostituição acessadas pelo estudo apresentaram uma taxa de 44 (58,7\%) para o não uso de preservativo com clientes. Este fato vem demonstrar que as mulheres em situação de prostituição nesta cidade, como em outras cidades do país, estão em uma situação de vulnerabilidade extrema, sendo essa explicada pelas desigualdades e pobreza da região acrescida do enfraquecimento das políticas públicas voltadas a populações de "risco" (Grangeiro, Castanheira \& Nemes, 2015).

Ressalta-se, ainda, que essa prática não deve ser associada à falta de preservativos já que 73 (97,3\%) afirmaram receber preservativos no local de trabalho. $\mathrm{O}$ acesso facilitado aos insumos de prevenção é extremamente importante para as mulheres em situação de prostituição que recebem um baixo preço pelo programa, situação das profissionais do sexo em muitos estudos brasileiros (Cunha; Moreira; Lôbo, 2012). Desta forma as mulheres não têm que optar entre comprar o preservativo, ou levar para casa o dinheiro recebido pelo programa para manutenção da família.

Evidenciou-se durante a realização deste estudo, embora não tenha sido objeto de investigação, outro fator de risco associado ao não uso de preservativos, onde percebeu-se a frequente utilização de bebidas alcoólicas e/ou drogas ilícitas sendo um fato concreto neste estudo. O efeito de drogas ilícitas, como maconha, cocaína e crack, ou lícitas, como cigarro e álcool, quando se está dominada pelas drogas, se prevenir torna-se algo mais complexo (Moura et al., 2010).

Outra fonte de risco de DST/HIV, considerada uma das principais é a recusa dos clientes em utilizar preservativos durante toda a relação sexual. A maioria das mulheres em situação de prostituição somente faz o programa se o cliente aceitar usar a camisinha, porém, durante o ato sexual, ele fica insistindo para retirar o preservativo. Essa atitude coloca em risco a saúde das mulheres e lhes causa sofrimento (Silva, Costa \& Nascimento, 2010). Aquino, Ximenes e Pinheiro (2010) consideram um dos grandes desafios enfrentados pelas mulheres em situação de prostituição está relacionado ao convencimento do cliente para o uso do preservativo. De um lado existe a resistência dele quanto à adoção de práticas seguras e, do outro, a fragilidade da profissionais do sexo ao lidar com tal situação.

Como a prostituição é um meio de sobrevivência, fica mais difícil resistir à pressão do cliente para realizar o programa sem o preservativo, já que percebemos que o não uso da camisinha implica, muitas vezes, um adicional aos preços do programa.

Embora $40(53,3 \%)$ das mulheres afirmem usar preservativos em todos os programas, a metade do total $38(50,7 \%)$ deixam de usá-lo com o parceiro fixo nessa perspectiva, mostra-se outra fonte de risco, que é o fato da mulheres em situação de prostituição aceitarem ter relações sexuais sem preservativo com parceiro fixo. Os resultados obtidos acerca da não utilização do preservativo com o parceiro fixo denotam a vulnerabilidade a que as mulheres pesquisadas estão submetidas. A média nacional de utilização do preservativo na relação com o companheiro é de 20\%. (Aquino, Ximenes \& Pinheiro, 2010).

Segundo Sousa (2013) esta relação de afetividade de acordo com alguns autores, cria um imaginário que se domina como "proteção imaginária". A crença nesta falsa proteção leva os sujeitos a não aderir ao uso do preservativo com estes parceiros "estáveis" ou a realizar uma avaliação de risco e concluir que este tipo de parceiro não oferece risco para adquirir 
uma DST/HIV. Esta situação é confirmada com os dados deste estudo multicêntrico realizado no Brasil onde a maioria das profissionais do sexo expressa que aceitariam fazer sexo com parceiro fixo sem preservativo (Szwarcwald, 2010).

Percebemos que as estratégias de prevenção utilizadas pelas mulheres em situação de prostituição são diferenciadas de acordo com a natureza do vínculo com o parceiro sexual. Quando há algo considerado íntimo ou amoroso, o não uso do preservativo é visto como símbolo de fidelidade, enquanto a camisinha denota desconfiança.

Há necessidade de Estratégias de promoção à saúde entre profissionais do sexo incluindo mais acesso a insumos fundamentais ao seu trabalho: preservativos masculinos e femininos, lubrificantes (que evitam rompimento de preservativos principalmente no sexo anal), tratamentos emergenciais de DST (uso de bisturis elétricos no tratamento de condilomas), possibilitar acesso a consultas, exames e medicamentos. E serviços específicos em "áreas chave", com horários alternativos e estratégias de redução de risco sexual, além de estímulo ao seu protagonismo (Figueiredo \& Peixoto, 2010; Martins, 2015).

\section{Conclusão}

Este estudo revela que as mulheres em situação de prostituição do município de Sobral- CE vivem em uma situação de vida bastante desfavorável, considerando que uma alta proporção são solteiras ou separadas, jovens com média de idade de 26,7 anos, cobram baixo valor pelo programa, vivem apenas da prostituição, possuem baixo nível de escolaridade e baixa renda mensal. Estudo tem mostrado que estes fatores contribuem para a manutenção das práticas sexuais de risco, seja com clientes ou mesmos parceiros sexuais regulares.

O presente estudo mostra que as mulheres em situação de prostituição têm conhecimento adequado sobre a prevenção de IST/HIV, pois $100 \%$ das mulheres responderam que o preservativo masculino é um método que evita a IST/HIV. Contrastando com este resultado, observou-se uma atitude e prática inadequadas, pois mais da metade respondeu que aceitariam realizar programas sem preservativos por um valor mais alto e não utilizam preservativos com parceiros fixos e cliente preferenciais. E isto mostra que estas mulheres estão em um processo crescente de vulnerabilidades para IST/HIV.

A intervenção educativa desenvolvida por enfermeiras com enfoque na prevenção das IST/HIV promoveu efeitos desejáveis na medida em que se obteve melhora significativa em todos os itens do conhecimento e atitude, confirma-se a proposta de que uma intervenção de Enfermagem baseada em estratégia educativa, tendo seu conteúdo subsidiado pelos referenciais que ajudem na prevenção dos comportamentos de risco das profissionais do sexo, como os utilizados no presente estudo, Política Nacional de Atenção Integral à Saúde da Mulher (Brasil, 2004) e nas recomendações contidas nos Cadernos da Atenção Básica Caderno No 18 HIV/Aids, Hepatites e outras DST (Brasil, 2006), Caderno $N^{\circ} 13$ Controle dos Cânceres do Colo do Útero e da Mama (Brasil, 2013) do Ministério da Saúde, eficazes na melhoria do conhecimento e atitude das mulheres em situação de prostituição com relação a prevenção das IST/HIV.

Dentre as limitações do estudo pode-se destacar o curto intervalo de tempo entre a intervenção e a aplicação do pósteste, o que pode ter influenciado nos resultados desejáveis. Apesar desse possível viés, a melhoria de todos os itens do inquérito CAP mostra claramente que um projeto dessa natureza tem grande possibilidade de atingir a finalidade da prevenção das IST/HIV em mulheres profissionais do sexo, indo além do modelo tradicional de uma ação de saúde, mas refletido em suas vidas e no contexto social em que estão inseridas. Daí a importância da inserção das equipes de saúde da família junto a essas mulheres, por meio de estratégias educativas, defendendo e garantindo os direitos de saúde desse grupo populacional que ainda se encontram a margem da sociedade.

A relevância do desenvolvimento de estratégias educativas, como as realizadas nesta investigação, que abordem o conhecimento, atitude e prática na prevenção das IST/HIV, pelos profissionais de saúde, notadamente o enfermeiro, por meio das metodologias participativas, estratégias que permitem uma maior conscientização e influenciam positivamente nas 
mudanças de comportamento, induzindo a uma melhor condição de saúde. Dentre esses métodos, enfatiza-se o emprego de dinâmicas de grupo e oficinas, que proporcionam um sentimento de valorização, equidade e inclusão das participantes.

As estratégias educativas foram desenvolvidas em cinco encontros nas casas de prostituição, com estratégias pedagógicas participativas apoiadas por referenciais freirianos, adotando atividades específicas de educação em saúde, específicas para a promoção da saúde e a prevenção de doenças, sendo observado um processo de intensas trocas de experiências entre pessoas distintas e intercâmbios entre saberes, edificando, assim, o conhecimento e vínculo entre as participantes, podendo se configurar uma estratégia potente para reforçar o empoderamento.

\section{Agradecimentos}

À Associação Sobralense das Trabalhadoras do Sexo - ASTRA por articular os espaços e aproximação com as mulheres participantes deste estudo; à Secretaria de Saúde de Sobral por meio da COAS pelos insumos e apoio nas intervenções educativas, à Pró-Reitoria de Extensão e Cultura da UVA, pela assessoria pedagógica e apoio na certificação do curso.

\section{Referências}

Aquino, O. S., Ximenes, L. B., \& Pinheiro, A. K. B. (2010). Políticas públicas de saúde voltadas à atenção à prostituta: breve resgate histórico. Enfermagem em Foco, 1(1):18-22.

Brasil. (2003). Ministério da Saúde. Avaliação da efetividade das ações de prevenção dirigidas às profissionais do sexo, em três regiões brasileiras. Brasília: Ministério da Saúde, 2003.

Brasil. (2004). Ministério da Saúde. Política Nacional de Atenção à Saúde da Mulher: princípios e diretrizes. Brasília: Ministério da Saúde.

Brasil. (2005). Ministério da Saúde. Comportamento sexual da população brasileira e percepções do HIV/Aids. Brasília: Ministério da Saúde.

Brasil. (2006). Ministério da Saúde. HIV/Aids, hepatites e outras DST. Brasília: Ministério da Saúde.

Brasil. (2008). Ministério da Saúde. Manual de Prevenção das DST/HIV/Aids em Comunidades Populares. Secretaria de Vigilância Brasília: Ministério da Saúde, 2008.

Brasil. (2013). Ministério da Saúde. Controle dos cânceres do colo do útero e da mama. Brasília: Ministério da Saúde.

Brasil. (2020). Ministério da Saúde. Boletim Epidemiológico. Ministério da Saúde.

Cunha, J. X. P., Moreira, M. A. S. P., \& Lôbo, M. P. (2012). Women's vulnerability to HIV/AIDS: a systematic review. Journal of Nursing UFPE, 6(4), 874882 .

Escosteguy, C.C. Estudos de intervenção. (2009). Epidemiologia. Editora Atheneu.

Fernandes, R. L. V., Moura, E. R. F., Feitoza, A. R., Evangelista, D. R., \& Oriá, M. O. B. (2012). Conhecimento, atitude e prática relacionados ao preservativo feminino. Rev Rene, 13(4), 755-765.

Figueiredo, R., \& Peixoto, M. (2010). Profissionais do sexo e vulnerabilidade. Boletim do Instituto de Saúde (Impresso), 12(2), $196-201$.

Grangeiro, A., Castanheira, E. R., \& Nemes, M. I. B. (2015). A re-emergência da epidemia de aids no Brasil: desafios e perspectivas para o seu enfrentamento. Interface Comun. Saúde Educ., 19(52), 5-6.

Instituto Brasileiro de Geografia e Estatística. (2010). Indicadores demográficos. IBGE.

Martins, T.A. (2015). Prevalência e fatores associados à testagem para HIV entre as mulheres profissionais do sexo em Fortaleza. Tese de doutorado, Universidade Federal do Ceará/ Universidade Estadual do Ceará/ Universidade de Fortaleza, Fortaleza, CE, Brasil.

Matos, M. A. D., Caetano, K. A. A., França, D. D. D. S., Pinheiro, R. S., Moraes, L. C. D., \& Teles, S. A. (2013). Vulnerabilidade às Doenças Sexualmente Transmissíveis em mulheres que comercializam sexo em rota de prostituição e turismo sexual na Região Central do Brasil. Revista Latino-Americana de Enfermagem, 21(4), 906-912.

Moura, A. D. A., Oliveira, R. M. S. D., Lima, G. G. D., Farias, L. M., \& Feitoza, A. R. (2010). O comportamento de prostitutas em tempos de aids e outras doenças sexualmente transmissíveis: como estão se prevenindo? Texto \& Contexto-Enfermagem, 19(3), 545-553.

Moura, S. L. O., Silva, M. A. M, Moreira, A. C. A, Freitas, C. A. S. L, \& Pinheiro, A. K. B. (2021). Vulnerabilidade de mulheres às Infecções Sexualmente Transmissiveis. Esc Anna Nery, 25(1), 1-8. 
Nascimento, V. B., do Nascimento Martins, N. V., Ciosak, S. I., Nichiata, L. Y. I., dos Santos Oliveira, J. S., Bezerra, L. O., \& dos Santos, L. A. (2017). Vulnerabilidades de mulheres quilombolas no interior da Amazônia às infecções sexualmente transmissíveis: um relato de experiência. IJHE-Interdisciplinary Journal of Health Education, 2(1), 68-73.

Neri, É. D. A. R., Moura, M. S. S. D., Penha, J. C. D., Reis, T. G. O. D., Aquino, P. D. S., \& Pinheiro, A. K. B. (2013). Conhecimento, atitude e prática sobre o exame Papanicolaou de prostitutas. Texto \& Contexto-Enfermagem, 22(3), 731-738.

Nicolau, A. I. O., Ribeiro, S. G., Lessa, P. R. A., Monte, A. S., Ferreira, R. D. C. D. N., \& Pinheiro, A. K. B. (2012). A picture of the socioeconomic and sexual reality of women prisoners. Acta Paulista de Enfermagem, 25(3), 386-392.

Nunes, E. L. G. \& Andrade, A. G. (2009). Adolescentes em situação de rua: prostituição, drogas e HIV/aids em Santo André, Brasil. Psicol Soc., 21(1): 45-54.

Pogetto, B. D., Rodrigues, M., Silva, M. G., \& Parada, C. M. G. D. L. (2011). Prevalência de doenças sexualmente transmissíveis em mulheres profissionais do sexo, em um município do interior paulista, Brasil. Revista Latino-Americana de Enfermagem, 19, 493-499.

Polit, D.F. \& Beck, C.T. (2011). Fundamentos de pesquisa de enfermagem: avaliação de evidencias para a prática da enfermagem. (7a ed.), Artmed.

Salmeron, N.A. \& Pessoa, T.A.M. (2012). Profissionais do sexo: perfil socioepidemiológico e medidas de redução de danos. Acta paul. enferm, 25(4), 549554.

Santos, N. J., Barbosa, R. M., Pinho, A. A., Villela, W. V., Aidar, T., \& Filipe, E. (2009). Contextos de vulnerabilidade para o HIV entre mulheres brasileiras. Cadernos de Saúde Pública, 25(2), S321-S333.

Silitonga, N., Davies, S. C., Kaldor, J., Wignall, S., \& Okoseray, M. (2011). Prevalence over time and risk factors for sexually transmissible infections among newly-arrived female sex workers in Timika, Indonesia. Sexual health, 8(1), 61-64.

Silva, F. E., Costa, B. D., \& Nascimento, U.J. (2010). O trabalho das profissionais do sexo em diferentes lócus de prostituição da cidade. Psicologia: Teoria e Prática, 12(1), 109-122.

Sousa, R. M. R. B. (2013). Não sei direito como é, eu só sei que é AIDS: incentivos e barreiras ao teste de HIV/AIDS entre profissionais do sexo. Tese de doutorado, Universidade Federal do Ceará/ Universidade Estadual do Ceará/ Universidade de Fortaleza, Associação de IES- Ampla Associação, Fortaleza, CE, Brasil.

Szwarcwald, C. L. (2009). Taxas de prevalência de HIV e sífilis e conhecimento, atitudes e práticas de risco relacionadas às infecções sexualmente transmissíveis no grupo das mulheres profissionais do sexo, no Brasil. Relatório de pesquisa. Brasília: Ministério da Saúde.

Todd, C. S., Nasir, A., Stanekzai, M. R., Bautista, C. T., Botros, B. A., Scott, P. T., \& Tjaden, J. (2010). HIV, hepatitis B, and hepatitis C prevalence and associated risk behaviors among female sex workers in three Afghan cities. AIDS (London, England), 24(2), 69-75.

Vieira, M. S. (2015). Deslocamentos femininos e prostituição. Revista Estudos Feministas, 23(2), 629-632. 